\section{Christoph Bachmann}

\section{Echinacea}

Echinacea purpurea L., der Sonnenhut (Roter Sonnenhut, Purpursonnenhut), ist ein Vertreter der Gattung Echinacea aus der Familie der Asteraceen. Die Pflanze stammt ursprünglich aus Nordamerika, wo sie von den indigenen Völkern für verschiedene Anwendungen als Arzneipflanze eingesetzt wurde. In der ersten Hälfte des 20. Jahrhunderts wurde die Arzneipflanze nach Europa gebracht, wo sie seither kultiviert wird. Neben der Spezies «purpurea» werden auch die Arten Echinacea angustifolia sowie Echinacea pallida medizinisch verwendet. Die verschiedenen Echinaforce-Extrakte sind ethanolische Auszüge aus den Wurzeln von E. purpurea. Ein anderes Echinacea-Präparat wird als Presssaft des ganzen Krautes in den Handel gebracht.

$\mathrm{Zu}$ den wirksamkeitsbestimmenden Inhaltstoffen von E. purpurea gehören in erster Linie Alkylamide, aber auch ätherische Öle, Glykoproteine sowie Kaffeesäurederivate.

Zahlreiche pharmakologische und klinische Studien belegen die Wirksamkeit von Echinacea-Extrakten zur Behandlung und Prävention von Influenza (siehe z.B. [2-4]). Der grossen Anzahl von positiven Studien steht ein Cochrane-Review gegenüber [5], der die Wirksamkeit von Echinacea infrage stellt. Es ist aber bekannt, dass sich die Cochrane Collaboration der Phytotherapie gegenüber sehr kritisch verhält und über verschiedene pflanzliche Arzneimittel Reviews publiziert hat, die deren Wirksamkeit weitge-

\title{
Echinaforce Hotdrink ist Oseltamivir ebenbürtig: Eine klinische Studie beweist die Nichtunterlegenheit
}

Im Jahr 2015 wurde eine klinische Studie mit Echinaforce Hotdrink, einer neuartigen Zubereitung des Echinacea-Extraktes Echinaforce, publiziert [1]. Darin wurde der Echinaforce Hotdrink mit dem Neuraminidase-Hemmer Oseltamivir verglichen, der als Goldstandard der Influenza-Behandlung gilt. Dieser Vergleich dokumentiert die Nichtunterlegenheit des Hotdrinks gegenüber Oseltamivir bei der Behandlung von Influenza. Im nachfolgenden Artikel wird diese Studie vorgestellt.

hend infrage stellen, obwohl eine grosse Reihe von positiven Studien das Gegenteil belegt.

Echinaforce Hotdrink (in der Folge Hotdrink genannt) ist ein Spissumextrakt, der aus den Tinkturen des frischen Krautes (DEV 1:12, Auszugsmittel Ethanol 65\%) und der frischen Wurzel von E. purpurea (DEV 1:11, Auszugsmittel Ethanol 65\%) hergestellt und als Heissgetränk angewendet wird.

\section{Oseltamivir}

Oseltamivir ist ein antiviraler Wirkstoff, der neben anderen, ähnlichen Substanzen zur Klasse der Neuraminidase-Hemmer gehört. Diese hemmen die Vermehrung von Viren und können so die Dauer und den Schweregrad einer viral bedingten Influenza vermindern. Wie ihr Name sagt, hem- men sie bei Influenza-A- und - $\mathrm{B}-\mathrm{Vi}$ ren das Enzym Neuraminidase, das für die Freisetzung neu gebildeter Viren aus den Wirtszellen verantwortlich ist. Wichtig für eine effektive Wirksamkeit ist der möglichst frühe Beginn der Behandlung [6].

\section{Studiendesign}

Die hier beschriebene Studie wurde als randomisierte, doppelblinde Doppel-Dummy- und ParallelgruppenStudie angelegt und hatte zum Ziel, die Wirksamkeit und Sicherheit des Hotdrinks zur Behandlung akuter Influenza-Symptome mit Oseltamivir zu vergleichen.

Die in die Studie eingeschlossenen Probanden mit einer diagnostizierten akuten Influenza begannen so früh wie möglich mit der Behandlung. Dafür nahmen sie während 10 Tagen entwe-
Tab. 1. Schema der Dosierung

\begin{tabular}{lll}
\hline Tag & Hotdrink-Gruppe & Oseltamivir-Gruppe \\
\hline $1-5$ & $3 \times 5 \mathrm{ml}$ Hotdrink Verum & $2 \times 1$ Oseltamivir Verum \\
& $2 \times 1$ Oseltamivir Placebo & $3 \times 5 \mathrm{ml}$ Hotdrink Placebo \\
$6-10$ & $3 \times 5 \mathrm{ml}$ Hotdrink Verum & $2 \times 1$ Oseltamivir Placebo \\
& $2 \times 1$ Oseltamivir Placebo & $3 \times 5 \mathrm{ml}$ Hotdrink Placebo
\end{tabular}

\section{KARGER}

(C) 2017 S. Karger GmbH, Freiburg 
der dreimal täglich $5 \mathrm{ml}$ Hotdrink (in etwa $150 \mathrm{ml}$ heissem Wasser) oder während 5 Tagen zweimal täglich 1 Tablette Oseltamivir und weitere 5 Tage zweimal täglich eine Oseltamivir-Placebotablette ein.

Die Hotdrink-Gruppe nahm gleichzeitig während 10 Tagen zweimal täglich 1 Oseltamivir-Placebotablette ein. Die Oseltamivir-Gruppe nahm neben der beschriebenen Dosierung während 10 Tagen auch ein Hotdrink-Placebo ein (Tab. 1).

Alle Probanden führten ein Symptome-Tagebuch und notierten dort während der Studie oder bis zur Genesung am Morgen und am Abend die beobachteten Grippesymptome. Dazu gehörten Husten, Schnupfen, Halsschmerzen, Müdigkeit, Kopfschmerzen, Gliederschmerzen, Fieber usw. Die Beurteilung wurde folgendermassen ausgedrückt: $0=$ abwesend; $1=$ mild; 2 = moderat; 3 = schwer.

Primärer Endpunkt der Studie war die Anzahl der Patienten, bei denen nach 1, nach 5 und nach 10 Tagen die Grippesymptome besser oder verschwunden waren. Als Wiedergesundung (Recovery) wurde der erste Tag definiert, an dem die Symptome abends nicht mehr vorhanden oder nur noch mild waren.

Als sekundäre Endpunkte galten weitere Grippesymptome wie Körpertemperatur, Schlafstörungen, der Bedarf an erlaubter Komedikation, Arbeitsfähigkeit/Arbeitsunfähigkeit sowie Komplikationen in Zusammenhang mit der Influenza.

\section{Probanden}

Gemäss Studiendesign wurden Personen mit einer diagnostizierten Influenza in die Studie aufgenommen und möglichst früh mit den Studienpräparaten behandelt. Es wurden folgende Ausschlusskriterien definiert:

- Antibiotische/antivirale Therapie innerhalb des letzten Monats vor Studienbeginn.
Tab. 2. Symptome, die bei Baseline von den Probanden als moderat oder schwer bezeichnet wurden

\begin{tabular}{lll}
\hline Symptom & $\begin{array}{l}\text { Oseltamivir- } \\
\text { Gruppe, \% }\end{array}$ & $\begin{array}{l}\text { Hotdrink- } \\
\text { Gruppe, \% }\end{array}$ \\
\hline Fieberhaftigkeit & 69,03 & 69,27 \\
Husten & 76,15 & 82,82 \\
Kopfschmerzen & 54,31 & 67,19 \\
Müdigkeit & 75,64 & 80,21 \\
Gliederschmerzen & 58,88 & 70,31 \\
Halsschmerzen & 48,22 & 44,79 \\
Unwohlsein & 20,82 & 20,84 \\
Schnupfen & 59,39 & 66,67 \\
Hitze- und Kältegefühl & 61,42 & 59,16 \\
\hline
\end{tabular}

- Grippeimpfung innerhalb der letzten 12 Monate.

- Eine vermutete bakterielle Infektion.

- Bronchitis.

- Behandlung mit Steroiden oder immunsuppressiven Substanzen.

- Schwangerschaft/Stillzeit.

- Schwerwiegende Erkrankungen wie chronische Herzbeschwerden, Asthma, Diabetes usw.

Total wurden 473 Probanden mit frühen Grippesymptomen in die Studie aufgenommen. Davon konnten 203 in der Hotdrink-Gruppe sowie 217 in der Oseltamivir-Gruppe ausgewertet werden. Ihr Alter bewegte sich zwischen 12 und 70 Jahren. Ihre demographischen Daten wiesen keine statistisch relevanten Unterschiede auf.

Auch bei den aufgetretenen Grippesymptomen konnte bei den beiden Behandlungsgruppen kein wesentlicher Unterschied festgestellt werden.

Tabelle 2 zeigt die von den Patienten als moderat oder schwer bezeichneten Symptome.

Der Patientenflow und die Dropouts wurden dokumentiert.

Alle Resultate wurden statistisch ausgewertet.

\section{Resultate}

Tabelle 3 zeigt die Auswertung in Bezug auf die Wiedergesundung der beiden Behandlungsgruppen nach 1 , nach 5 und nach 10 Tagen sowie für den ganzen Behandlungszeitraum. Weiterhin wird die Nichtunterlegenheit des Hotdrinks gegenüber Oseltamivir in Bezug auf die Wiedergesundung gezeigt. Bei den Therapieversagern nach 10 Tagen konnte eine nichtsignifikante Überlegenheit des Hotdrinks gegenüber Oseltamivir festgestellt werden:

- Therapieversager Hotdrink: 9,9\%.

- Therapieversager Oseltamivir: $15,2 \%$.

\section{Subgruppenauswertungen}

Nachweisbarkeit von Influenzaviren

Als eine der Subgruppenauswertungen wurde ermittelt, bei welcher Prozentzahl der Probanden nach 1, nach 5 und nach 10 Tagen im Nasenschleim keine Influenza-A- oder -B-Viren mehr nachweisbar waren (Tab. 4):

Diese Zahlen belegen wiederum die Nichtunterlegenheit des Hotdrinks verglichen mit Oseltamivir. Im Gegenteil: Am Tag 10 zeigte sich eine signifikante Überlegenheit des Hotdrinks ( $p=0,0365)$.

\section{Grippesymptome}

Als weitere Subgruppenauswertung wurde in beiden Studiengruppen das Verschwinden der individuellen Grippesymptome beobachtet. In beiden Behandlungsgruppen verschwanden die individuellen Symptome rasch, ohne dass zwischen den beiden Behandlungsgruppen ein Unterschied feststellbar war. Beim Verschwinden der Hauptsymptome einer Influenza (Husten, Kopfschmerzen, Gliederschmerzen) wurde eine nichtsignifikante Überlegenheit ( $p$ > 0,05 ) für die Hotdrink-Gruppe beobachtet. In Bezug auf die Normalisierung der Körpertemperatur (Fieberfreiheit innerhalb von 2 Tagen), der Wiedererlangung der Arbeitsfähigkeit und der Schlafstörungen liessen sich zwischen den beiden Behandlungsgruppen keine Unterschiede feststellen. 
Tab. 3. Wiedergesundung

\begin{tabular}{|c|c|c|c|c|c|c|c|c|}
\hline & \multicolumn{2}{|c|}{ Nach 1 Tag } & \multicolumn{2}{|c|}{ Nach 5 Tagen } & \multicolumn{2}{|c|}{ Nach 10 Tagen } & \multicolumn{2}{|l|}{ Total } \\
\hline & Hotdrink & Oseltamivir & Hotdrink & Oseltamivir & Hotdrink & Oseltamivir & Hotdrink & Oseltamivir \\
\hline Wiedergesundung, \% & 1,5 & 4,1 & 50,2 & 48,8 & 90,1 & 84,8 & & \\
\hline Mann-Whitney-Statistik & \multirow{2}{*}{\multicolumn{2}{|c|}{0,4867}} & \multirow{2}{*}{\multicolumn{2}{|c|}{0,5070}} & \multirow{2}{*}{\multicolumn{2}{|c|}{0,5268}} & \multicolumn{2}{|r|}{0,5068} \\
\hline Konfidenzintervall & & & & & & & \multicolumn{2}{|c|}{$0,48871-0,52265$} \\
\hline
\end{tabular}

\section{Komplikationen}

Bei der Anzahl der festgestellten Komplikationen (respiratorische und gastrointestinale) zeigte sich ein Vorteil für die Hotdrink-Gruppe, der nahe der Signifikanz war $(p=0,079)$. In beiden Behandlungsgruppen wurden nur bei $1,8 \%$ (Oseltamivir) bzw. 2,0\% (Hotdrink) der Probanden Antibiotika eingesetzt.

Bei der Verwendung von zusätzlichen Medikamenten konnte zwischen den Behandlungsgruppen kein statistisch signifikanter Unterschied festgestellt werden.

\section{Beurteilung der Behandlung}

Als gut bis sehr gut wurden die vorgenommenen Behandlungen von Prüfärzten und Probanden folgendermassen (\% aller Beteiligter) beurteilt:

- Oseltamivir: Prüfärzte: 91,2\%; Probanden: $86,2 \%$.

- Hotdrink: Prüfärzte: 91,6\%; Probanden: $87,2 \%$.

\section{Sicherheit}

26 Patienten (11,4\%) der HotdrinkGruppe berichteten von 31 sowie 32 Patienten der Oseltamivir-Gruppe von 44 unerwünschten Ereignissen. 10 dieser unerwünschten Ereignisse wurden in Zusammenhang mit den Studienmedikamenten in Zusammenhang

Tab. 4. Nachweisbarkeit von Influenza-A- oder -B-Viren im Nasenschleim nach 1, 5 und 10 Tagen

\begin{tabular}{llllllll}
\hline Nach 1 Tag, \% & & \multicolumn{2}{ll}{ Nach 5 Tagen, \% } & & \multicolumn{2}{l}{ Nach 10 Tagen, \% } \\
\cline { 1 - 3 } \cline { 5 - 6 } Hotdrink & Oseltamivir & & Hotdrink & Oseltamivir & & Hotdrink & Oseltamivir \\
\hline 0 & 0 & 45,0 & 42,9 & 95,0 & 76,2 \\
\hline
\end{tabular}

gebracht, 4 davon in der HotdrinkGruppe sowie 6 in der Vergleichsgruppe. Dies führte bei 5 Probanden in der Hotdrink-Gruppe bzw. 12 in der Oseltamivir-Gruppe zum Behandlungsstopp. Gastrointestinale Beschwerden traten in der OseltamivirGruppe fünfmal häufiger auf als in der Hotdrink-Gruppe. Es wurde jedoch von keinen schwerwiegenden unerwünschten Ereignissen berichtet, und auch die Laborparameter blieben innerhalb der Norm.

In beiden Behandlungsgruppen wurde die Verträglichkeit der Studienpräparate von über $90 \%$ der Probanden $(95,6 \%$ Hotdrink; 93\% Oseltamivir) als gut bis sehr gut bezeichnet.

\section{Diskussion}

Der Echinaforce Hotdrink stellt eine neue Einnahmeform von E. purpurea dar. In der hier beschriebenen Studie wurde seine Wirksamkeit und Sicherheit bei der Behandlung von Influenza A und B mit dem Goldstandard Oseltamivir verglichen. Das Studiendesign entsprach früheren Studi- en, welche die Überlegenheit von Oseltamivir gegenüber Placebo dokumentierten. Die Probanden wurden durchschnittlich nach $25,1 \mathrm{~h}$ nach Auftreten der ersten Symptome in die Studie aufgenommen. Unter diesen Bedingungen wurde die Nichtunterlegenheit von Echinaforce Hotdrink gegenüber Oseltamivir in Bezug auf die Wiedergenesung dokumentiert.

Trotz leicht stärkerer Symptome bei Baseline erreichten die Symptome nach 2-3 Tagen gleiche Werte. Am Ende der Behandlung waren in der Hotdrink-Gruppe mehr Patienten genesen als in der Vergleichsgruppe.

\section{Fazit}

Der Echinaforce Hotdrink zeigte sich in dieser Studie verglichen mit dem Neuraminidase-Hemmer Oseltamivir als gleichwertig und in Bezug auf die Sicherheit als überlegen. Damit empfiehlt sich dieses OTC-Präparat als wirksame und sichere Behandlung beginnend im Frühstadium einer Influenza.

\section{Literatur}

1 Rauš K, Pleschka S, Klein P, Schoop R, Fisher P: Effect of an Echinacea-based hot drink versus oseltamivir in influenza treatment: a randomized, double-blind, double-dummy, multicenter, noninferiority clinical trial. Curr Ther Res Clin Exp 2015;77:66-72.

2 Jawad M, Schoop R, Suter A, Klein P, Eccles R: Safety and efficacy profile of Echinacea purpurea to prevent common cold episodes: a randomized, double-blind, placebo-controlled trial. Evid Based Complement Alternat Med 2012;2012:841315.
3 Shah SA, Sander S, White CM, Rinaldi M, Coleman CI: Evaluation of echinaceae for the prevention and treatment of the common cold: a meta-analysis. Lancet Infect Dis 2007;7: 473-480. 
4 Pleschka S, Stein M, Schoop R, Hudson JB: Anti-viral properties and mode of action of standardized Echinacea purpurea extract against highly pathogenic avian influenza virus (H5N1, H7N7) and swine-origin $\mathrm{H} 1 \mathrm{~N} 1$ (S-OIV). Virol J 2009;6:197-206.
5 Karsch-Völk M, Barrett B, Kiefer D, Bauer R, Ardjomand-Woelkart K, Linde K: Echinacea for preventing and treating the common cold. Cochrane Database Syst Rev 2014;(2):CD000530.
6 Treanor J, Hayden FG, Vrooman PS, Barbarash R, Bettis R, Riff D, Singh S, Kinnersley N, Ward P, Mills RG: Efficacy and safety of the oral neuraminidase inhibitor oseltamivir in treating acute influenza: a randomized controlled trial. US Oral Neuraminidase Study Group. JAMA 2000;283:1016-1024. 